\title{
Inverse design of nonequilibrium steady states: A large-deviation approach
}

\author{
William D. Piñeros $\oplus^{1}$ and Tsvi Tlusty $\oplus^{1,2, *}$ \\ ${ }^{1}$ Center for Soft and Living Matter, Institute for Basic Science (IBS), Ulsan 44919, Republic of Korea \\ ${ }^{2}$ Department of Physics and Department of Chemistry, Ulsan National Institute of Science and Technology (UNIST), \\ Ulsan 44919, Republic of Korea
}

(Received 16 October 2020; accepted 12 January 2021; published 1 February 2021)

\begin{abstract}
The design of small scale nonequilibrium steady states (NESS) is a challenging, open ended question. While similar equilibrium problems are tractable using standard thermodynamics, a generalized description for nonequilibrium systems is lacking, making the design problem particularly difficult. Here we show we can exploit the large-deviation behavior of a Brownian particle and design a variety of geometrically complex steady-state density distributions and flux field flows. We achieve this design target from direct knowledge of the joint large-deviation functional for the empirical density and flow, and a "relaxation" algorithm on the desired target states via adjustable force field parameters. We validate the method by replicating analytical results, and demonstrate its capacity to yield complex prescribed targets, such as rose-curve or polygonal shapes on the plane. We consider this dynamical fluctuation approach a first step towards the design of more complex NESS where general frameworks are otherwise still lacking.
\end{abstract}

DOI: 10.1103/PhysRevE.103.022101

\section{INTRODUCTION}

The ability to create and design a system to a desirable goal stands as the culmination of deliberate human control and precise, applied knowledge. While this level of achievement is generally possible at comparative macroscales where bulk properties dominate, similar control is lacking for systems below the microscale where fluctuations, thermal or otherwise, is significant. Notwithstanding, the obvious appreciation of biological complexity and its myriad of small-scale solutions to practical problems [1,2], for instance, suggests the design barrier is not insurmountable. Indeed, design problems involving small systems have been an active topic of research in soft or condensed materials [3-5], motivated by the rising synthetic and technological prowess at this scale [6-9].

Broadly, one can envision the design problem as a generic optimization goal where one seeks to fulfill an objective subject to constraints that reflect the desired state of a system. Solution follows through standard numerical optimizers where possible. Such formulations can also be heuristic [10,11], or inferential, e.g., learning algorithms [12-14], and are practical in a variety of systems insofar as they can yield an answer [15-17]. However, in framing the design problem, it is desirable to maintain a clear physical picture so as to better understand the nature of the solution and its optimization process. For instance, in the equilibrium case, one usually seeks to minimize a free energy of a system, for example by modifying the underlying interactions $[18,19]$. Indeed, such an approach can be applied successfully to the design of a variety of self-assembling phases [20-23].

Extending a similar approach to the problem of design of nonequilibrium systems at the small scale is a critical yet mostly unaddressed issue. While a fundamental frame- work akin equilibrium thermodynamics remains elusive, some general statements at the dynamical fluctuations level such as fluctuation theorems are known [24-28] and experimentally demonstrated [29-31]. In particular, to make a nonequilibrium problem more tractable, one generally restricts attention to steady states, which are time invariant and therefore amenable to averaging. Critically, what distinguishes a nonequilibrium steady state (NESS) from an equilibrium one is the presence of nonvanishing fluxes. Therefore, in addition to the usual probability measure of a system's configuration, a viable statistical description must also account for the system's fluxes.

In this regard, the theory of large deviations can fill the gap and provide a window into a NESS system from a dynamical level. Chiefly, the large-deviations framework concerns the deviation of a quantity from its most likely expectation in the limit of infinite sampling. Specifically, a stochastic quantity $x$ fulfills a large-deviation principle if its probability measure scales as $P(x) \approx \exp [-n I(x)]$ in the large-sample limit, ${ }^{1}$ where $n$ is the number of samples, and $I(x)$ is a so-called rate function [32,33]. The most likely value of $x$ is therefore the minimum of the rate function.

Importantly, rate functions provide a compact statistical representation of a general stochastic process, where information about the average and variances is readily available. Indeed, large-deviation principles are applicable for equilibrium systems in the large-particle limit where rate functions enter in the form of ensemble-specific expressions. For instance, in the canonical ensemble the rate function takes the role of a free energy, and the equilibrium state is recovered as a constrained minimum of this rate function (the most likely state) [34]. Thus, knowledge of a rate function can provide a practical statistical description of a system's asymptotic 
behavior without the detailed tracking of a complex dynamics. For a rigorous definition of rate functions and technical treatment of large deviations, we refer to existing literature [35-37].

Here we concern ourselves with a NESS dynamics for which a large-deviation principle exists, such that information about its configurations and flux is available via a rate function. In particular, we consider the paradigmatic case of a colloidal particle in a viscous bath whose dynamics can be modeled through an overdamped Langevin equation:

$$
\dot{\mathbf{x}}=\chi \mathbf{f}(\mathbf{x})+(2 \chi \beta)^{-1 / 2} \boldsymbol{\xi}(t),
$$

where $\dot{\mathbf{x}}$ is the velocity vector of a particle, $\mathbf{x}$ its position, $\mathbf{f}(\mathbf{x})$ are forces, $\chi$ is the mobility, $\beta=1 / k_{\mathrm{B}} T$ is the inverse bath temperature ( $k_{\mathrm{B}}$ set to unity hereafter), and $\xi$ is a standard white-noise term. Non-equilibrium is achieved by means of nonconservative forces applied on the particle.

For these type of Markovian diffusion systems, the fluctuations can be captured as a joint probability of configurations and fluxes $[38,39]$, in the form of a rate functional:

$$
I[\rho, \mathbf{j}]=\frac{\beta}{4} \int d \mathbf{x} \rho(\mathbf{x})\left[\frac{\mathbf{j}(\mathbf{x})}{\rho(\mathbf{x})}-\frac{\mathbf{j}_{\rho}(\mathbf{x})}{\rho(\mathbf{x})}\right]^{2},
$$

where $\mathbf{j}_{\rho}(\mathbf{x})$ is given by

$$
\frac{\mathbf{j}_{\rho}(\mathbf{x})}{\rho(\mathbf{x})}=\frac{\mathbf{j}_{\mathrm{st}}(\mathbf{x})}{p_{\mathrm{st}}(\mathbf{x})}-\frac{1}{\beta} \nabla \ln \frac{\rho(\mathbf{x})}{p_{\mathrm{st}}(\mathbf{x})} .
$$

In Eqs. (2) and (3), $p_{\text {st }}$ is the most probable steady-state occupation probability, and the function $\rho(\mathbf{x})$ is an empirical density of spatial configurations defined as the time average over a trajectory $\mathbf{x}(t)$ during time period $\mathcal{T}$ as

$$
\rho(\mathbf{y} ; \mathbf{x})=\frac{1}{\mathcal{T}} \int_{0}^{\mathcal{T}} d t \delta(\mathbf{y}-\mathbf{x}) .
$$

Similarly, $\mathbf{j}_{\text {st }}(\mathbf{x})$ is the steady-state flux and $\mathbf{j}(\mathbf{x})$ represents the empirical flux or current defined as

$$
\mathbf{j}(\mathbf{y} ; \mathbf{x})=\frac{1}{\mathcal{T}} \int_{0}^{\mathcal{T}} d t \dot{\mathbf{x}}(t) \delta(\mathbf{y}-\mathbf{x}),
$$

where the integral is taken in the Stratonovich sense. In short, these empirical measures are finite-time averages of the system, where the true densities are achieved in the long-time limit. Altogether, the rate functional $I$ in Eq. (2) reflects the most relevant information on the expected steady-state system, in terms of its configuration density and flux.

In this work, we exploit knowledge of the Brownian particle's rate functional, and show we can leverage the dynamical fluctuations to design nonequilibrium steady states. Specifically, we imagine a target state as a far-off fluctuation that, through adjustment of appropriate force field parameters $\boldsymbol{\theta}$, "relaxes" as the most probable steady state, i.e., minimizes the rate function. We carry out this parameter update directly from a Brownian simulation, given a prescribed form of the configuration and flux configuration that represents a steady-state target. We remark that this approach differs from numerous force field fitting studies of Brownian systems, where the goal is to replicate known trajectories or experimental data, and are usually done at a statistical inference level [40-45]. Instead, here we focus on creating arbitrary NESS in the form of specific configuration probabilities and fluxes, which we do through direct knowledge of the system's dynamic behavior. As we will elaborate below, this method can recover known analytical answers and help directly realize nontrivial steady states.

The rest of the paper is structured as follows. We elaborate on the mathematical and simulation details of our method in Sec. II. In Sec. III we first validate our method against a simple analytical scenario. We then show we can realize various prescribed NESS featuring complex occupation probabilities and fluxes in the shape of rose-curve and polygon motifs. We also offer some thoughts on a physical interpretation of the method. We end with some conclusions and offer some possible future directions in Sec. IV.

\section{METHODS}

\section{A. General design approach}

We approach the nonequilibrium steady-state design problem from a fluctuation dynamics point of view, using the rate functional in Eq. (2). We envision the steady-state target as a far-off fluctuation that is "relaxed" via adjustments of a force field parameters $\boldsymbol{\theta}$ so as to become the most probable steady state, i.e., $\rho(\mathbf{x})=p_{\text {st }}(\mathbf{x})$ and $\mathbf{j}(\mathbf{x})=\mathbf{j}_{\text {st }}(\mathbf{x})$. This requires that the rate function be minimized relative to parameters such that $\nabla_{\theta} I=0$ and therefore $I=0$. We demonstrate this goal can be achieved via an iterative gradient descent method on the parameters by minimization of the rate functional as follows.

First we rewrite the rate functional in Eq. (2), using Eq. (3), as

$$
\begin{aligned}
I[\rho, \mathbf{j}]= & \frac{\beta}{4} \int d \mathbf{x} \rho(\mathbf{x})\left[\frac{1}{\beta} \nabla \ln \frac{\rho(\mathbf{x})}{p_{\mathrm{st}}(\mathbf{x})}\right]^{2} \\
& +\frac{\beta}{4} \int d \mathbf{x} \rho(\mathbf{x})\left[\frac{\mathbf{j}(\mathbf{x})}{\rho(\mathbf{x})}-\frac{\mathbf{j}_{\mathrm{st}}(\mathbf{x})}{p_{\mathrm{st}}(\mathbf{x})}\right]^{2} \\
\equiv & I_{A}[\rho]+I_{B}[\rho, \mathbf{j}],
\end{aligned}
$$

where $I_{A}$ depends on probability $\rho(\mathbf{x})$ alone and $I_{B}$ contains the flux field dependent terms. We identify the fluctuations $\mathbf{j}(\mathbf{x})$ and $\rho(\mathbf{x})$ as prescribing the target state, and let $p_{\text {st }}(\mathbf{x} \mid \boldsymbol{\theta})$ and $\mathbf{j}_{\mathrm{st}}(\mathbf{x} \mid \boldsymbol{\theta})$ be intermediate steady states controlled through $\boldsymbol{\theta}$.

We can now update parameters $\boldsymbol{\theta}$ via a standard iterative gradient descent procedure as

$$
\boldsymbol{\theta}^{i+1}=\boldsymbol{\theta}^{i}-\alpha_{A} \nabla_{\boldsymbol{\theta}^{i}} I_{A}\left(\boldsymbol{\theta}^{i}\right)-\alpha_{B} \nabla_{\boldsymbol{\theta}^{i}} I_{B}\left(\boldsymbol{\theta}^{i}\right),
$$

where $\boldsymbol{\theta}^{i}$ indicates vector of parameters at iteration $i$, and $\alpha_{A}$ and $\alpha_{B}$ are the respective gradient update step sizes. The parameter gradients themselves can be evaluated as

$$
\begin{aligned}
& \nabla_{\boldsymbol{\theta}^{i}} I_{A}\left(\boldsymbol{\theta}^{i}\right) \\
& \quad=-\frac{1}{2 \beta} \int d \mathbf{x} \rho(\mathbf{x})\left[\nabla \ln \frac{\rho(\mathbf{x})}{p_{\mathrm{st}}\left(\mathbf{x} \mid \boldsymbol{\theta}^{i}\right)}\right] \cdot \nabla_{\boldsymbol{\theta}^{i}} \nabla \ln p_{\mathrm{st}}\left(\mathbf{x} \mid \boldsymbol{\theta}^{i}\right)
\end{aligned}
$$

and

$$
\begin{aligned}
& \nabla_{\boldsymbol{\theta}^{i}} I_{B}\left(\boldsymbol{\theta}^{i}\right) \\
& \quad=\frac{\beta}{2} \int d \mathbf{x} \rho(\mathbf{x})\left[\frac{\mathbf{j}(\mathbf{x})}{\rho(\mathbf{x})}-\frac{\mathbf{j}_{\mathrm{st}}\left(\mathbf{x} \mid \boldsymbol{\theta}^{i}\right)}{p_{\mathrm{st}}\left(\mathbf{x} \mid \boldsymbol{\theta}^{i}\right)}\right] \cdot \nabla_{\boldsymbol{\theta}^{i}}\left(\frac{\mathbf{j}_{\mathrm{st}}\left(\mathbf{x} \mid \boldsymbol{\theta}^{i}\right)}{p_{\mathrm{st}}\left(\mathbf{x} \mid \boldsymbol{\theta}^{i}\right)}\right) .
\end{aligned}
$$



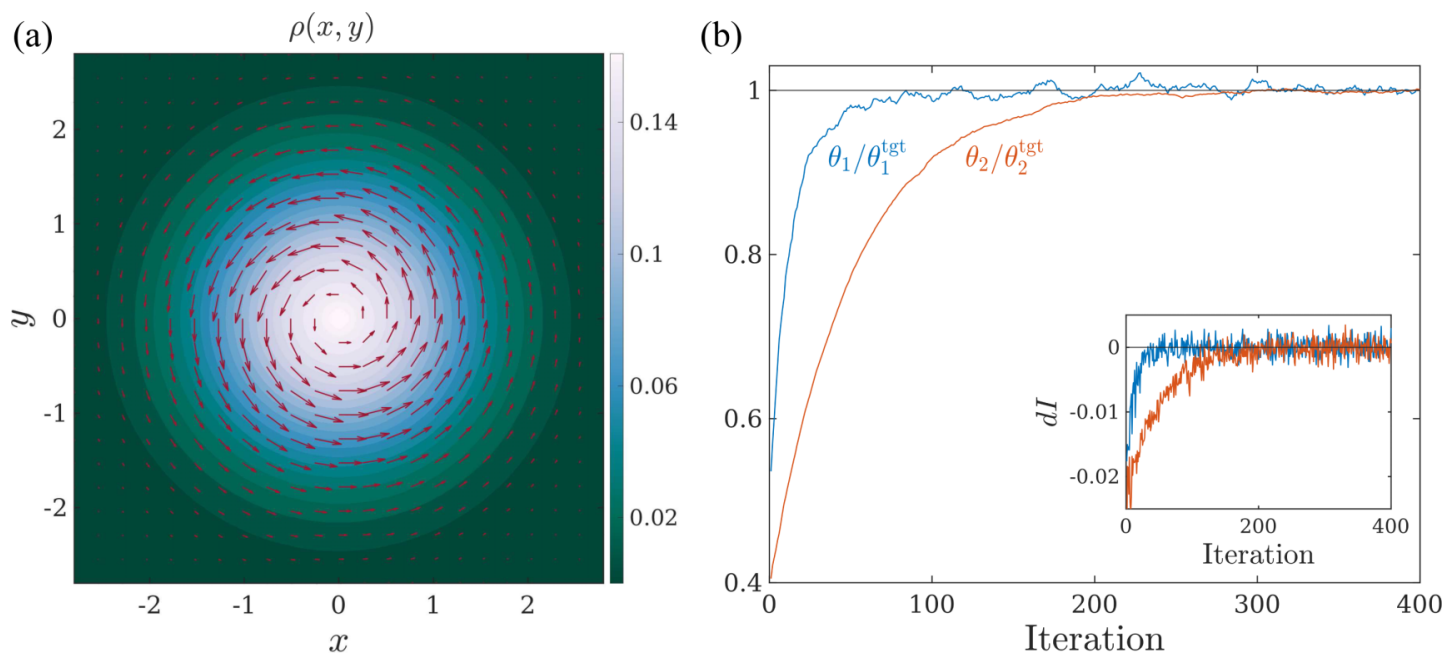

FIG. 1. (a) Test case: a particle in a harmonic potential with a driven angular field whose respective strengths are controlled by parameters $\theta_{i}$ [Eq. (14)]. Analytic solution is given by a Gaussian probability and an angular flux [Eq. (15)]. (b) Progression of the parameters along the iterative gradient descent procedure, for targets defined as the steady-state solution of Eq. (14) with $\beta=2, \theta_{1}^{\text {tgt }}=0.5, \theta_{2}^{\text {tgt }}=2$, where parameters $\theta_{i}$ are randomly initiated. Solution is achieved when $\theta_{i} / \theta_{i}^{\text {tgt }}=1$, corresponding to $d I \equiv \nabla_{\theta} I_{A / B} \rightarrow 0$ (inset).

The effective steady-state velocity $\mathbf{j}_{\mathrm{st}}\left(\mathbf{x} \mid \boldsymbol{\theta}^{i}\right) / p_{\mathrm{st}}\left(\mathbf{x} \mid \boldsymbol{\theta}^{i}\right)$ in Eq. (9) can be computed from the Fokker-Planck expression $\mathbf{j}_{\mathrm{st}} / p_{\mathrm{st}}=$ $\mathbf{f}-\beta^{-1} \nabla \ln p_{\mathrm{st}}$ as

$$
\nabla_{\boldsymbol{\theta}^{i}}\left(\frac{\mathbf{j}_{\mathrm{st}}\left(\mathbf{x} \mid \boldsymbol{\theta}^{i}\right)}{p_{\mathrm{st}}\left(\mathbf{x} \mid \boldsymbol{\theta}^{i}\right)}\right)=\nabla_{\boldsymbol{\theta}^{\mathbf{i}}} \mathbf{f}\left(\mathbf{x} \mid \boldsymbol{\theta}^{i}\right)-\beta^{-1} \nabla_{\boldsymbol{\theta}^{i}} \ln p_{\mathrm{st}}\left(\mathbf{x} \mid \boldsymbol{\theta}^{i}\right)
$$

Each of the integrals in Eqs. (8) and (9) can be evaluated with knowledge of the fixed target states $[\rho(\mathbf{x}), \mathbf{j}(\mathbf{x})]$, adjustable force field $\mathbf{f}\left(\boldsymbol{\theta}^{i}\right)$, and intermediate steady states $p_{\text {st }}^{i}$, the latter which are found directly from a Brownian simulation.

The optimization procedure is then carried out in the following fashion: (A) given an initial guess of the force field $\mathbf{f}\left(\boldsymbol{\theta}^{i}\right)$ compute $p_{\mathrm{st}}^{i}$ from a Brownian simulation; (B) Use $p_{\mathrm{st}}^{i}$ to evaluate gradients in Eqs. (8) and (9); (C) update parameters as per Eq. (7); (D) repeat iteration using updated $\mathbf{f}\left(\boldsymbol{\theta}^{i+1}\right)$. The optimization then completes when gradients converge to zero.

As a model of an adjustable force field $\mathbf{f}$ (parameters left implicit), we consider

$$
\mathbf{f}=\mathbf{f}_{h}+\mathbf{f}_{a n}+\mathbf{f}_{o}+\mathbf{f}_{s},
$$

where $\mathbf{f}_{h}$ is a confining harmonic force, $\mathbf{f}_{a n}$ is a driven angular force, $\mathbf{f}_{o}$ is an isotropic repulsive term at the origin, and $\mathbf{f}_{s}$ are a series of variable sinusoidal terms. Thus, the system can be interpreted as a confined particle subject to complex sinusoidal forces which constrain it to a specific occupation shape and flow. For further details on model implementation, Brownian simulation, force field choices and parameter solutions, see the Supplemental Material [46].

\section{B. Design targets}

Having defined the design strategy, we now consider concrete design targets that highlight the efficacy of the procedure. To this end, we consider a two-dimensional colloidal particle such that its average occupation and flow trace a specific figure motif on the plane. As a first example, we consider a family of "rose" curves resembling flower petals:

$$
r_{c}(\phi)=r_{0}[1-a \cos (k \phi)],
$$

where $r_{c}$ is the radial coordinate, $\phi$ is the polar angle, $r_{0}$ is the size of the petals, $k$ is the number of petals, and $a \in(0,1]$ controls petal sharpness. An illustration is provided in the inset of Fig. 2(a) below. Target probability density is then defined as a normalized Gaussian-like function,

$$
p_{\text {tgt }}(r, \phi)=\frac{1}{N} \exp \left[-\frac{\left[r-r_{c}(\phi)\right]^{2}}{2 \sigma^{2}}\right],
$$

where $N$ is the normalization factor, and $r_{c}$ is defined by the rose curve in Eq. (12).

Defining a target flux field $\mathbf{j}_{\text {tgt }}$ is somewhat more challenging since, by definition, $\nabla \cdot \mathbf{j}_{\mathrm{tgt}}=0$. Thus, creating a vanishing divergence is nontrivial, and instead we approximate the flux field as a flow tangential to the chosen motif figure. $^{2}$ As we will see, this procedure ensures that the pre-

\footnotetext{
${ }^{2}$ Of course, exact knowledge of both the density and flux field would define the force field through the Fokker-Planck expression for the flux. However, in such a case, the method's purpose would still lie in finding a simpler, closed-form force model from otherwise fully prescriptive targets.
}

TABLE I. Design target construction parameters. For the rosemotif target, these correspond to $k$, the number of petals, and $a$, the petal sharpness. For all roses, $r_{0}=1.25$ is the petal size and $\sigma^{2}=0.5$

\begin{tabular}{|c|c|c|}
\hline & Roses & Polygons \\
\hline 1 & $k=3 ; a=0.4$ & hexagon; $l=1.25, \quad \sigma^{2}=0.30$ \\
\hline 2 & $k=4 ; a=0.4$ & square; $l=1$, \\
\hline 3 & $k=12 ; a=0.3$ & triangle; $l=1.20, \quad \sigma^{2}=0.45$ \\
\hline
\end{tabular}
is the variance of the Gaussian-like function. For polygons, $l$ is the side-length size for the respective equilateral polygon centered at the origin, and $\sigma^{2}$ is the variance of the brush Gaussian profile (see text). 

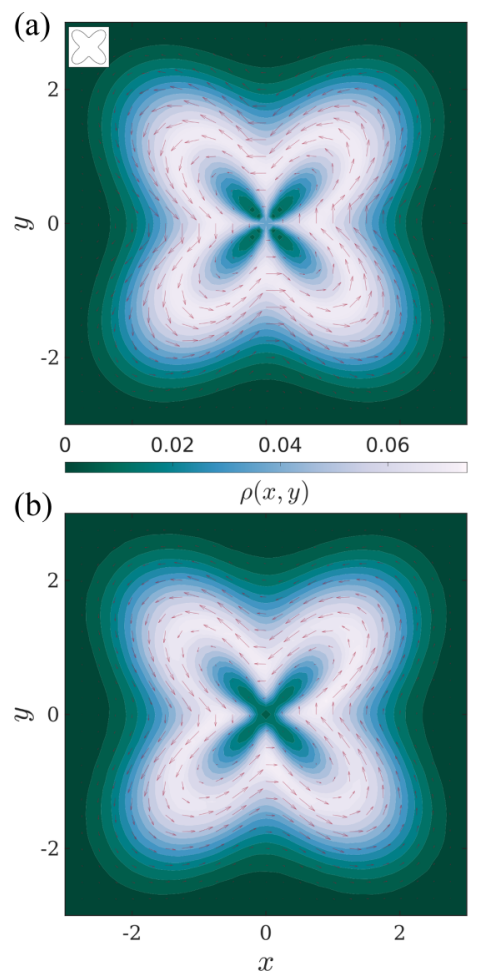

(e)

(d)

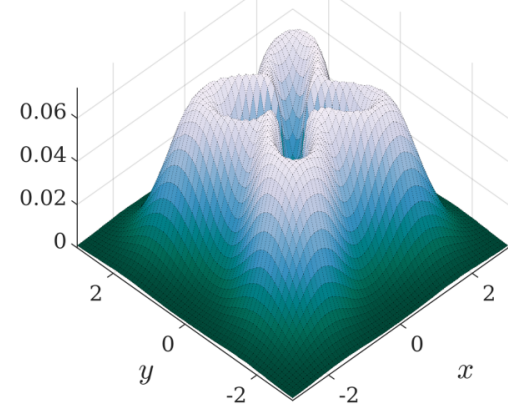

(f)
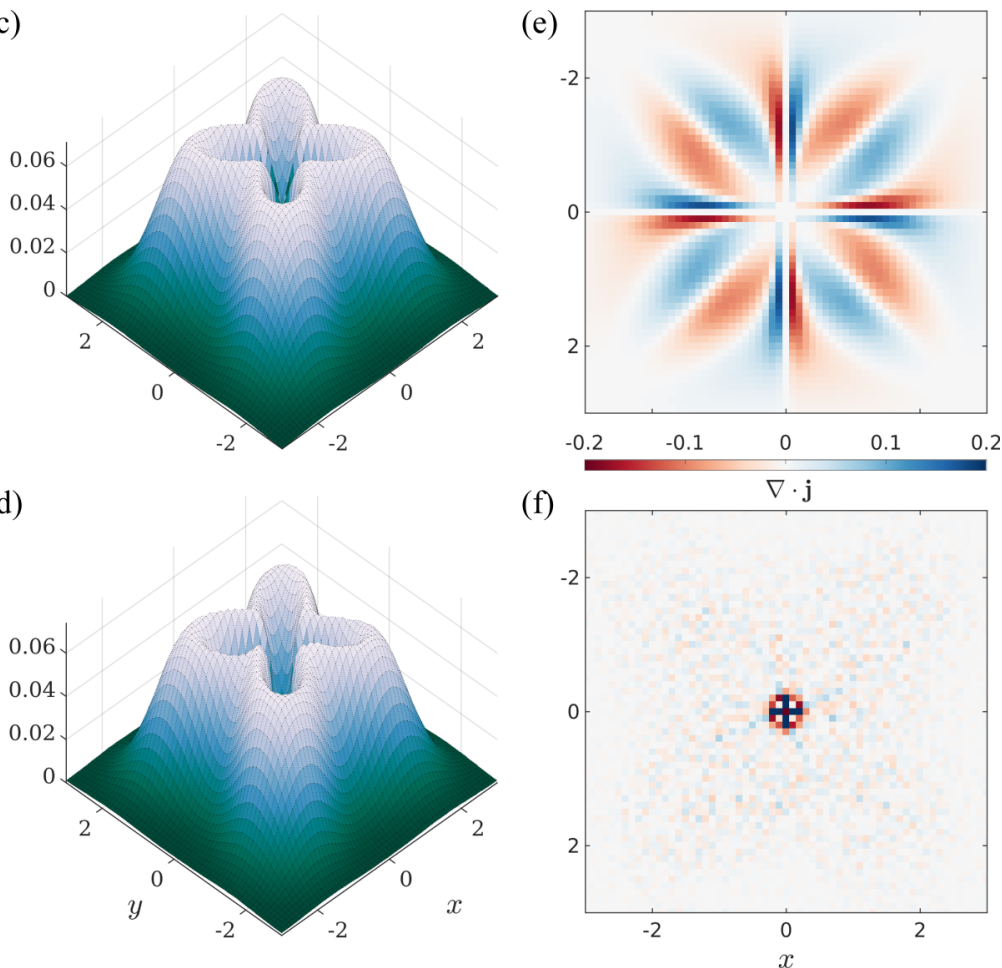

FIG. 2. Representative example of a rose-curve target steady state $(k=4)$. (a) Target probability density $\rho(x, y)$ and flux field (red arrows). (b) Realized target probability density and flux. Small inset is the parametric rose-curve plot [Eq. (12)] with $r_{0}=1.25, k=4$, and $a=0.4$. (c),(d) Perspective views of (a) and (b) respectively. (e) Divergence of the flux $\nabla \cdot \mathbf{j}$ for the input target and the realized result in (f). While the input is divergent, the realized result from a simulation obtains a valid flux field by default. Nonzero values in (f) are a finite grid size artifact.

scribed flux field remains plausible as a realizable target. Final chosen target parameters are shown in Table I.

Additionally, we consider a second family of more challenging design targets in the form of polygonal motifs. The probability $p_{\text {tgt }}(r, \phi)$ is now piecewise continuous and drawn as a polygon brush whose size is scaled linearly along the plane, and its paint intensity is determined by an angular symmetric Gaussian profile $\exp \left[-\left(s-s_{0}\right)^{2} / 2 \sigma^{2}\right]$, where $s$ is the scaling and $s_{0}=1$. As in the rose-curve case, the flux field is chosen as a uniform flow tangent to the polygon shape. Chosen polygonal targets are equilateral triangle, square, and hexagon with parameters as shown in Table I.

\section{RESULTS AND DISCUSSION}

We begin by proof testing the method outlined in Sec. II. To this end, we consider the simple analytical case of a particle confined by a radial harmonic potential and driven by an angular force along the plane. The force field is

$$
\mathbf{f}=-\theta_{1} r \hat{\mathbf{r}}+\theta_{2} r \hat{\boldsymbol{\phi}}
$$

where the first term is a harmonic force with confining constant strength $\theta_{1}$, and the second is an angular force with driving magnitude $\theta_{2}$. The analytical steady-state solution of this system is known [38] [Fig. 1(a)]:

$$
p_{\mathrm{st}}(r)=\frac{\beta \theta_{1}}{2 \pi} \exp \left[-\frac{\beta \theta_{1}}{2} r^{2}\right], \quad \mathbf{j}_{\mathrm{st}}(r)=p_{\mathrm{st}}(r) \theta_{2} r \hat{\boldsymbol{\phi}},
$$

where $\beta$ is the inverse bath temperature. Thus, given a chosen set of $\boldsymbol{\theta}^{\text {tgt }}=\left\{\theta_{1}^{\text {tgt }}, \theta_{2}^{\text {tgt }}\right\}$, we know the resulting steady-state density $p_{\text {st }}$ and $\mathbf{j}_{\text {st }}$ which we can set as test design targets. Then, using the model force with tunable parameters $\boldsymbol{\theta}=$ $\left\{\theta_{1}, \theta_{2}\right\}$ in Eq. (14), we can check if the method we propose finds the prescribed $\boldsymbol{\theta}^{\text {tgt }}$ values. The results of this test are shown in Fig. 1(b), where the adjustable $\theta_{i}$ are initiated randomly and their convergence progress is monitored as a function of iteration number. As seen, the ratio of $\theta_{i} / \theta_{i}^{\text {tgt }}$ converges to unity past around 300 iterations, indicating successful parameter solution. This convergence corresponds to the vanishing of the rate functional gradients, $d I \equiv \nabla_{\theta} I_{A / B}$ (inset) indicating rate function minimization.

Next, we move on to consider more complex steady-state design targets. To this end, we first start with the rose-motif set of targets which, as elaborated in Sec. II B, represent particle confinement and flow constrained to trace out a flowerlike pattern along the plane. As a representative case, we consider the four-petal rose curve $(k=4)$ whose respective target $p_{\text {st }}$ and $\mathbf{j}_{\text {st }}$ as are illustrated in contour projection [Fig. 2(a)] and relief representation [Fig. 2(c)]. As seen in the second figure row in (b) and (d), our method successfully replicates the prescribed probability and flux field density with excellent agreement. Importantly, while the input target field is not entirely valid as seen by the nonzero divergence [Fig. 2(e)], the realized field not only approximates the desired design flow, it also fully realizable, i.e., divergence-free [Fig. 2(f)]. This follows directly from the simulation component of the method, where parameters can only be adjusted with knowledge of the intermediate state probability and flux field densities. Thus, the 

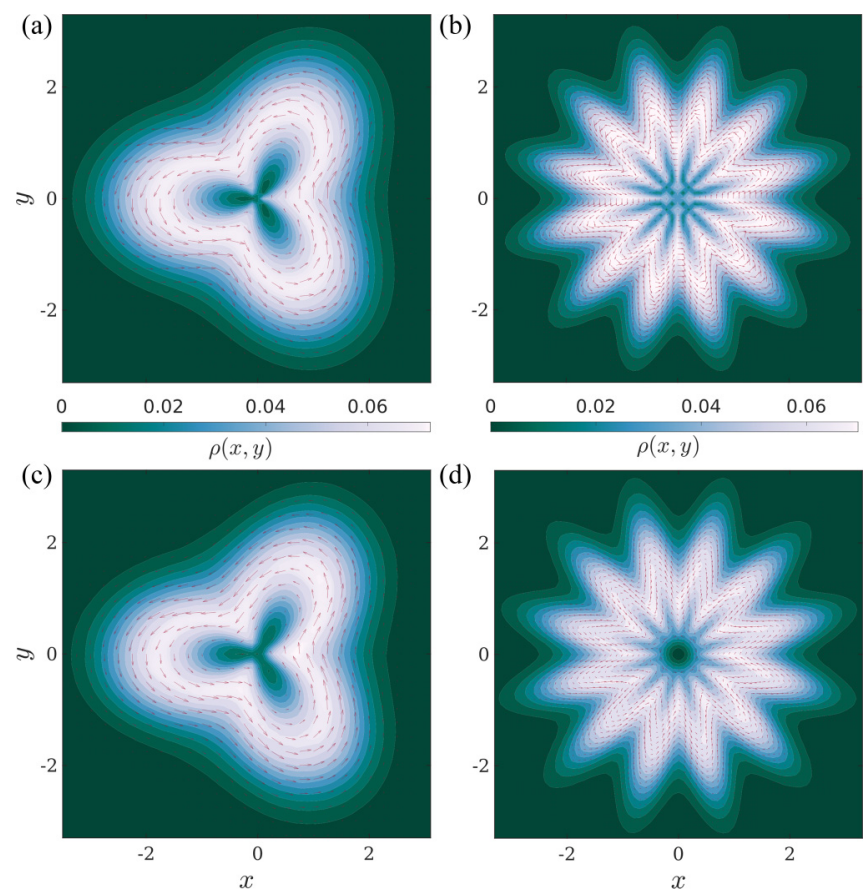

FIG. 3. Sample probability $\rho(x, y)$ and flux field targets (red arrows) using the rose-curve motif for (a) $k=3$ and (b) $k=12$. (c) and (d) are the respective realized solutions using our method.

difficulty of creating divergenceless flux fields as a design target can be averted by ensuring the target flux field is simply plausible within the motif constraint.
In Fig. 3, we show two additional examples of designed rose-motif targets for an odd number of petals $k=3$ in (a), and a more complex rose pattern with $k=12$ in (b). As seen in (c) and (d), both targets are successfully designed with both flux fields conforming to the imposed motif. Notably, in the case of $k=12$, the target displays rapidly changing flux fields with tight changes around every petal turn. Despite this, the generated flux field nicely conforms to figure and naturally smooths out the more jarring, and unrealizable, kinks in the original input target.

Next, we consider more challenging motifs tracing polygon-shaped densities and field fluxes. Unlike the smooth rose-motif targets, here piecewise continuous, rectilinear edges are imposed on the steady-state targets. Due to the singularities, one expects such hard-cut densities and flows to be only asymptotically realizable. Yet, assuming a sufficiently flexible force field is provided, we expect the method to find the best approximate realization. Indeed, as seen in Fig. 4, probability densities and flux fields displaying (a) hexagonal, (b) triangular, and (c) square motifs are realized. Notably, the difficulty in achieving such targets is observed in the solutions (d)-(f), where the hard-edged corners become rounded, and the rectilinear flows along the edges exhibit small deviations along the tracks. The triangle motif proved particularly difficult to solve, requiring larger sampling times, double phase terms, and additional sinusoidal angular driven terms. The difficulty is mostly due to the acute angles that impose stronger singularity and deviation from smoothness, as well as the reflection asymmetry along the $x$ axis.

We end with the following tentative thoughts and observations. While our method works well for the design of visually
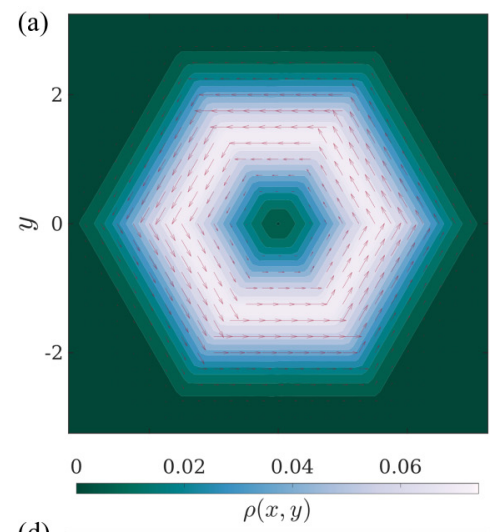

(d)

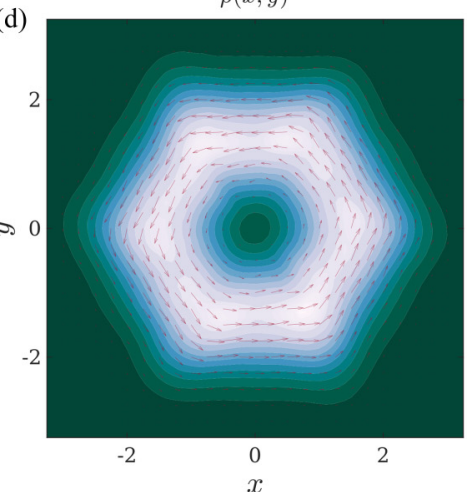

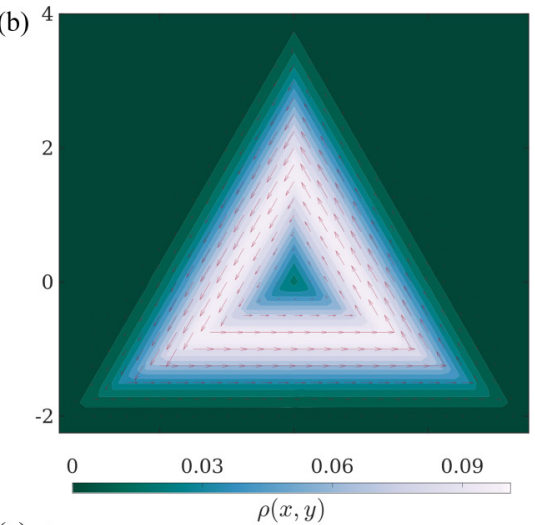

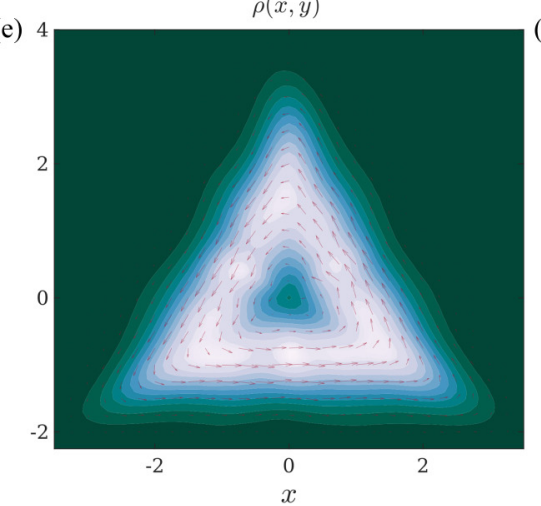

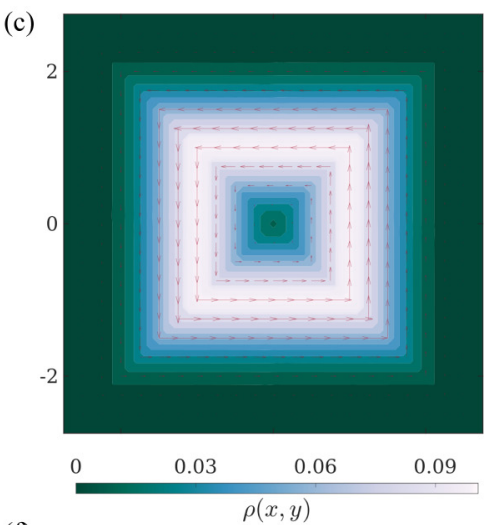

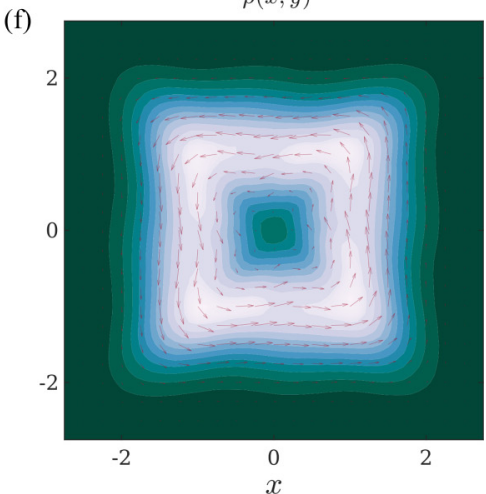

FIG. 4. Piecewise continuous targets with polygonal motifs in position probability density $\rho(x, y)$ and flux fields (red arrows). (a)-(c) Input targets for hexagon, triangle, and square polygon motifs, respectively. (d)-(f) Realized probability density $\rho(x, y)$ and flux fields (red arrows). 
appealing steady states, its practicality is limited to the explicit knowledge of a rate function of the relevant dynamics, such as interactionless Brownian particles. Extending this approach to the more interesting case of interparticle interactions relegates the problem to the establishment of an appropriate rate function which can be challenging outside of system approximations like mean-field interactions [36,47]. What is notable, however, is that it is indeed possible to (1) conceive a desirable target state as a dynamical fluctuation and (2) realize it as the most likely steady state following an appropriate parameter "relaxation."

This fluctuation-relaxation picture is, perhaps, not entirely surprising given its central role in the fluctuation-response theory for near-equilibrium processes, where the most likely relaxation pathway following a perturbation also corresponds to an equivalent spontaneous fluctuation pathway of the system $[48,49]$. In fact, it recently came to our attention that the rate function itself can be estimated tightly with a mapping of optimal target states through a variational procedure, consistent with a reciprocal picture [50]. Furthermore, this relationship between relaxation and fluctuation has been shown to hold for NESS Markovian processes in the hydrodynamic limit, supposing a corresponding large-deviation principle exists [51-53]. Indeed, the term $I_{A}(\rho)$ in the rate function Eq. (2) can be identified as a nonadiabatic entropy production term vanishing upon relaxation to the steady state [54,55]. Likewise, the rate functional can be related to an entropy flux and a quantity termed "traffic" (which is a form of dynamical activity), both of which play a central role in the fluctuationresponse theory for nonequilibrium diffusive systems [56,57]. Tentatively, from a NESS design point of view, all these observations suggest that a fluctuation level approach might be generally useful beyond exact knowledge of the corresponding dynamical rate function.

\section{CONCLUSION}

In this work, we leveraged dynamical fluctuations in an overdamped Brownian system to design a variety of nontrivial nonequilibrium steady states (NESS). In particular, we envisioned the target state as a far-off fluctuation that is "relaxed" to a desirable steady state by minimizing the known rate functional with respect to an adjustable force field parameters $\boldsymbol{\theta}$. We demonstrated that such an approach successfully replicates the analytic solution of a particle in a harmonic potential driven by an angular field, and applied it to the design of nontrivial steady states in the form of prescribed occupation probabilities and steady-state fluxes tracing rosecurve and polygon motifs. We showed all targets could be successfully achieved, including the polygon motifs which were expected to be more difficult due to the presence of sharp turns and hard-edge linear flow. Furthermore, satisfactory solution could be achieved even if the prescribed flux field was strictly unrealizable though still plausible for the motif constraint. Altogether, our method demonstrates that a dynamic fluctuation point of view provides a feasible entry into NESS design, though much work is needed in extending it to useful scenarios such as interacting particle ensembles. For future studies, it will be interesting to understand how this design view may tie into more general frameworks of nonequilibrium fluctuation response or established entropy production relations.

\section{ACKNOWLEDGMENTS}

The authors thank Daniel Nickelsen, Christian Maes, and Juzar Thingna for helpful discussions. This work was publicly funded through the Institute for Basic Science, South Korea, Project Code No. IBS-R020.
[1] K. Autumn, M. Sitti, Y. A. Liang, A. M. Peattie, W. R. Hansen, S. Sponberg, T. W. Kenny, R. Fearing, J. N. Israelachvili, and R. J. Full, Evidence for van der Waals adhesion in gecko setae, Proc. Natl. Acad. Sci. USA 99, 12252 (2002).

[2] A. B. Kolomeisky and M. E. Fisher, Molecular motors: A theorist's perspective, Annu. Rev. Phys. Chem. 58, 675 (2007).

[3] S. Molesky, Z. Lin, A. Y. Piggott, W. Jin, J. Vucković, and A. W. Rodriguez, Inverse design in nanophotonics, Nat. Photonics 12, 659 (2018).

[4] A. Zunger, Inverse design in search of materials with target functionalities, Nat. Rev. Chem. 2, 0121 (2018).

[5] V. Mlinar, Utilization of inverse approach in the design of materials over nano- to macro-scale, Ann. Phys. (NY) 527, 187 (2015).

[6] R. A. Hughes, E. Menumerov, and S. Neretina, When lithography meets self-assembly: A review of recent advances in the directed assembly of complex metal nanostructures on planar and textured surfaces, Nanotechnology 28, 282002 (2017).

[7] C. M. Soukoulis and M. Wegener, Past achievements and future challenges in the development of three-dimensional photonic metmamaterials, Nat. Photonics 5, 523 (2011).

[8] G. M. Whitesides and B. Grzybowski, Self-assembly at all scales, Science 295, 2418 (2002).

[9] M. A. Boles, M. Engel, and D. V. Talapin, Self-assembly of colloidal nanocrystals: From intricate structures to functional materials, Chem. Rev. 116, 11220 (2016).

[10] X.-S. Yang, Nature-inspired optimization algorithms: Challenges and open problems, J. Comput. Sci. 46, 101104 (2020).

[11] M. Z. Miskin, G. Khaira, J. J. de Pablo, and H. M. Jaeger, Turning statistical physics models into materials design engines, Proc. Natl. Acad. Sci. USA 113, 34 (2016).

[12] K. T. Butler, D. W. Davies, H. Cartwright, O. Isayev, and A. Walsh, Machine learning for molecular and materials science, Nature (London) 559, 547 (2018).

[13] A. L. Ferguson, Machine learning and data science in soft materials engineering, J. Phys.: Condens. Matter 30, 043002 (2017).

[14] N. E. Jackson, M. A. Webb, and J. J. de Pablo, Recent advances in machine learning towards multiscale soft materials design, Curr. Opin. Chem. Eng. 23, 106 (2019).

[15] K. Yue and K. A. Dill, Inverse protein folding problem: Designing polymer sequences, Proc. Natl. Acad. Sci. USA 89, 4163 (1992).

[16] J. Noh, J. Kim, H. S. Stein, B. Sanchez-Lengeling, J. M. Gregoire, A. Aspuru-Guzik, and Y. Jung, Inverse design of solid-state materials via a continuous representation, Matter 1, 1370 (2019). 
[17] V. Mlinar and A. Zunger, Spectral barcoding of quantum dots: Deciphering structural motifs from the excitonic spectra, Phys. Rev. B 80, 035328 (2009).

[18] S. Torquato, Inverse optimization techniques for targeted selfassembly, Soft Matter 5, 1157 (2009).

[19] Z. M. Sherman, M. P. Howard, B. A. Lindquist, R. B. Jadrich, and T. M. Truskett, Inverse methods for design of soft materials, J. Chem. Phys. 152, 140902 (2020).

[20] A. F. Hannon, Y. Ding, W. Bai, C. A. Ross, and A. Alexander-Katz, Optimizing topographical templates for directed self-assembly of block copolymers via inverse design simulations, Nano Lett. 14, 318 (2014).

[21] A. Jain, J. R. Errington, and T. M. Truskett, Dimensionality and Design of Isotropic Interactions that Stabilize Honeycomb, Square, Simple Cubic, and Diamond Lattices, Phys. Rev. X 4, 031049 (2014).

[22] W. D. Piñeros and T. M. Truskett, Designing pairwise interactions that stabilize open crystals: Truncated square and truncated hexagonal lattices, J. Chem. Phys. 146, 144501 (2017).

[23] N. A. Mahynski, R. Mao, E. Pretti, V. K. Shen, and J. Mittal, Grand canonical inverse design of multicomponent colloidal crystals, Soft Matter 16, 3187 (2020).

[24] R. J. Harris and G. M. Schütz, Fluctuation theorems for stochastic dynamics, J. Stat. Mech.: Theory Exp. (2007) P07020.

[25] D. J. Evans and D. J. Searles, The fluctuation theorem, Adv. Phys. 51, 1529 (2002).

[26] R. Chetrite and K. Gawędzki, Fluctuation relations for diffusion processes, Commun. Math. Phys. 282, 469 (2008).

[27] U. Seifert, Stochastic thermodynamics, fluctuation theorems and molecular machines, Rep. Prog. Phys. 75, 126001 (2012).

[28] U. Seifert, From stochastic thermodynamics to thermodynamic inference, Annu. Rev. Condens. Matter Phys. 10, 171 (2019).

[29] D. Collin, F. Ritort, C. Jarzynski, S. B. Smith, I. Tinoco, and C. Bustamante, Verification of the Crooks fluctuation theorem and recovery of RNA folding free energies, Nature (London) 437 , 231 (2005).

[30] V. Blickle, T. Speck, L. Helden, U. Seifert, and C. Bechinger, Thermodynamics of a Colloidal Particle in a Time-Dependent Nonharmonic Potential, Phys. Rev. Lett. 96, 070603 (2006).

[31] G. M. Wang, J. C. Reid, D. M. Carberry, D. R. M. Williams, E. M. Sevick, and D. J. Evans, Experimental study of the fluctuation theorem in a nonequilibrium steady state, Phys. Rev. E 71, 046142 (2005).

[32] B. Derrida, Non-equilibrium steady states: Fluctuations and large deviations of the density and of the current, J. Stat. Mech.: Theory Exp. (2007) P07023.

[33] A. C. Barato and R. Chetrite, A formal view on level 2.5 large deviations and fluctuation relations, J. Stat. Phys. 160, 1154 (2015).

[34] Y. Oono, Large deviation and statistical physics, Prog. Theor. Phys. Suppl. 99, 165 (1989).

[35] S. R. S. Varadhan, Large Deviations and Applications (Society for Industrial and Applied Mathematics, Philadelphia, 1984).

[36] H. Touchette, The large deviation approach to statistical mechanics, Phys. Rep. 478, 1 (2009).

[37] H. Touchette and R. J. Harris, Large deviation approach to nonequilibrium systems, in Nonequilibrium Statistical Physics of Small Systems (John Wiley \& Sons, New York, 2013), Chap. 11, pp. 335-360.
[38] J. Hoppenau, D. Nickelsen, and A. Engel, Level 2 and level 2.5 large deviation functionals for systems with and without detailed balance, New J. Phys. 18, 083010 (2016).

[39] C. Maes, K. Netočný, and B. Wynants, Steady state statistics of driven diffusions, Physica A 387, 2675 (2008).

[40] C. L. Vestergaard, Optimizing experimental parameters for tracking of diffusing particles, Phys. Rev. E 94, 022401 (2016).

[41] R. Sarfati, J. Bławzdziewicz, and E. R. Dufresne, Maximum likelihood estimations of force and mobility from single short Brownian trajectories, Soft Matter 13, 2174 (2017).

[42] L. Pérez García, J. Donlucas Pérez, G. Volpe, A. V. Arzola, and G. Volpe, High-performance reconstruction of microscopic force fields from Brownian trajectories, Nat. Commun. 9, 5166 (2018).

[43] M. E. Beheiry, M. Dahan, and J.-B. Masson, Inference MAP: mapping of single-molecule dynamics with Bayesian inference, Nat. Methods 12, 594 (2015).

[44] S. Thapa, M. A. Lomholt, J. Krog, A. G. Cherstvy, and R. Metzler, Bayesian analysis of single-particle tracking data using the nested-sampling algorithm: Maximum-likelihood model selection applied to stochastic-diffusivity data, Phys. Chem. Chem. Phys. 20, 29018 (2018).

[45] A. Frishman and P. Ronceray, Learning Force Fields from Stochastic Trajectories, Phys. Rev. X 10, 021009 (2020).

[46] See Supplemental Material at http://link.aps.org/supplemental/ 10.1103/PhysRevE.103.022101 for further implementation details and parameter solutions.

[47] C. Orrieri, Large deviations for interacting particle systems: Joint mean-field and small-noise limit, Electron. J. Probab. 25, 44 (2020).

[48] L. Onsager and S. Machlup, Fluctuations and irreversible processes, Phys. Rev. 91, 1505 (1953).

[49] R. Kubo, Response, relaxation and fluctuation, in Quantum Statistical Mechanics in the Natural Sciences: A Volume Dedicated to Lars Onsager on the Occasion of his Seventieth Birthday, edited by S. L. Mintz and S. M. Widmayer (Springer, Boston, 1974), pp. 299-326.

[50] S. Whitelam, D. Jacobson, and I. Tamblyn, Evolutionary reinforcement learning of dynamical large deviations, J. Chem. Phys. 153, 044113 (2020).

[51] L. Bertini, A. De Sole, D. Gabrielli, G. Jona-Lasinio, and C. Landim, Fluctuations in Stationary Nonequilibrium States of Irreversible Processes, Phys. Rev. Lett. 87, 040601 (2001).

[52] L. Bertini, A. De Sole, D. Gabrielli, G. Jona-Lasinio, and C. Landim, Minimum dissipation principle in stationary nonequilibrium states, J. Stat. Phys. 116, 831 (2004).

[53] L. Bertini, A. De Sole, D. Gabrielli, G. Jona-Lasinio, and C. Landim, Macroscopic fluctuation theory, Rev. Mod. Phys. 87, 593 (2015).

[54] M. Esposito, U. Harbola, and S. Mukamel, Entropy fluctuation theorems in driven open systems: Application to electron counting statistics, Phys. Rev. E 76, 031132 (2007).

[55] M. Esposito and C. Van den Broeck, Three Detailed Fluctuation Theorems, Phys. Rev. Lett. 104, 090601 (2010).

[56] C. Maes and M. H. van Wieren, Time-Symmetric Fluctuations in Nonequilibrium Systems, Phys. Rev. Lett. 96, 240601 (2006).

[57] M. Baiesi, C. Maes, and B. Wynants, Fluctuations and Response of Nonequilibrium States, Phys. Rev. Lett. 103, 010602 (2009). 